\title{
ID2 Inhibits Lung Adenocarcinoma Cell Malignant Behaviors Through Inhibiting the Activation of the $\mathrm{PI3K} / \mathrm{AKT} / \mathrm{mTOR}$ Signaling Pathway
}

\section{Wenzhong Peng}

Central South University

Jia Chen

third people's hospital of pingxiang city

\section{Ruoxi He}

Central South University

Yongjun Tang

Central South University

Juan Jiang

Central South University

Ying Li ( $\square$ Liying971@163.com )

Central South University https://orcid.org/0000-0003-0061-7062

\section{Research}

Keywords: lung cancer, lung adenocarcinoma cells, inhibitor of DNA binding 2 (ID2), the PI3K/AKT/mTOR signaling

Posted Date: September 27th, 2021

DOI: https://doi.org/10.21203/rs.3.rs-817478/v1

License: (c) (1) This work is licensed under a Creative Commons Attribution 4.0 International License. Read Full License 


\section{Abstract}

Background: Lung cancer is the most common cancer and one of the main causes of cancer-related deaths, and it manifests as metastatic disease in most cases. Considering frequent gene mutation and/or signaling deregulation in lung adenocarcinoma, identifying novel factors or agents targeting these signaling pathways might be promising strategies for lung adenocarcinoma therapy.

Methods: GEO datasets were analyzed to identify differentially expressed genes (DEGs) in lung adenocarcinoma. The specific effects of candidate gene overexpression or knockdown on lung adenocarcinoma cell phenotypes were examined. Gene Ontology (GO), Kyoto Encyclopedia of Genes and Genomes (KEGG) are used to connect the genomic and functional information of DEGs. The dynamic effects of candidate gene and signaling pathway agonist on lung adenocarcinoma malignant behaviors were investigated. Finally, clinical lung adenocarcinoma and adjacent non-cancerous tissues were collected and the levels of candidate gene were examined in tissue samples.

Results: Inhibitor of DNA binding 2 (ID2) was identified as an aberrantly downregulated gene in lung adenocarcinoma. ID2 overexpression suppressed lung adenocarcinoma cell viability, colony formation capacity, and migration. ID2 overexpression also reduced the protein levels of N-cadherin, MMP2, MMP9, and the phosphorylation of AKT and mTOR. The PI3K/AKT/mTOR signaling agonist exerted opposite effects on lung adenocarcinoma cells to those of ID2 overexpression, and partially reversed the effects of ID2 overexpression. In tissue samples, ID2 protein levels and mRNA expression were also downregulated compared with those in adjacent non-cancerous tissues.

Conclusion: ID2 exerts its tumor-suppressive effects on lung adenocarcinoma cell malignant behaviors through inhibiting the activation of the PI3K/AKT/mTOR signaling pathway. Restoring ID2 expression in lung adenocarcinoma cells might improve the curative effect of lung adenocarcinoma therapies.

\section{Introduction}

Lung cancer is the most common cancer and one of the main causes of cancer-related deaths, and it manifests as metastatic disease in most cases [1]. According to histology, lung cancer mainly includes two subtypes: small cell lung cancer and non-small-cell lung cancer (NSCLC), which account for $15 \%$ and $85 \%$ of all cases, respectively [2]. NSCLC could be further divided into three types: squamous cell carcinoma, adenocarcinoma, and large cell carcinoma. Since lung adenocarcinoma accounts for about $40 \%$ of all lung cancers, lung adenocarcinoma has been regarded as the most common type of lung cancer. Lung adenocarcinoma develops from small airway epithelial, type II alveolar cells, which secrete mucus and other substances [3, 4]. Gene mutations and focal amplification regulate cancer cell sensitivity to induced cell death. Thus, the difference in treatment sensitivity generally depends on the susceptibility of lung cancer cells, especially lung adenocarcinoma cells, to cell death [5].

The latest advances in the molecular map have identified multiple aberrations that participate in lung adenocarcinoma initiation and development, thus making personalized treatments possible. For example, 
the activation of WNT/TCF pathway is considered to be a determinant of brain and bone metastasis in the progression of lung adenocarcinoma. It shows that the gene expression characteristics indicating WNT/TCF activation are related to the multiple organ recurrence of primary lung adenocarcinoma [6]. By using a cancer cell invasion model and microarray analysis, Borczuk et al. [8] identified and characterized TGF- $ß$ signaling downstream mediators critical for lung adenocarcinoma invasion; in other words, type II transforming growth factor-beta receptor has been recognized as a repressor of lung adenocarcinoma invasiveness. Oncogene fusion in lung adenocarcinoma usually arises from complex genome rearrangements. in first-line treatment, detection of EGFR mutations is crucial, because EGFR tyrosine kinase inhibitors are able to target specific mutations in the EGFR gene, therefore improving the prognosis of patients with EGFR-mutant lung adenocarcinoma $[10,11]$. The driver fusion oncogene-generating rearrangements of lung adenocarcinoma, including EML4-ALK, CD74-ROS1, and KIF5B-RET [12] and other molecular-driven therapeutic targets may lead to the paradigm shift in advanced lung adenocarcinoma therapies. Moreover, subsequent activation of two major downstream cascades, RAS-RAF-MEK-ERK and PI3K-Akt-mTOR, would lead to the hyperproliferation of cancer cell and uncontrolled tumor growth. As one of the most commonly deregulated pathways, the PI3K-Akt-mTOR signaling has been reported to participate in the tumorigenesis of lung adenocarcinoma $[15,16]$. Identifying novel factors or agents targeting these signaling pathways might be promising strategies for lung adenocarcinoma therapy.

Microarray technology has increasingly become a promising tool for the study of oncology medicine, and comprehensive bioinformatics analyses allow mining tumor-related biomarkers from big data, contributing to diagnosing lung adenocarcinoma at early stages and lung adenocarcinoma prognosis evaluation [17-20]. For example, searching the public genome database such as Gene Expression Comprehensive Database (GEO) help to identify a series of cancer-related gene deregulation, as well as multiple-gene signature playing a potential role in lung adenocarcinoma diagnosis and prognosis [21]. By using data from The Cancer Genome Atlas (TCGA) database-lung adenocarcinoma, He et al. [23] identified 138 IncRNAs, upregulated or downregulated in lung adenocarcinoma, analyzed the expression correlation, and formed the IncRNA-mRNA co-expression network, providing new clues for these IncRNAs as diagnostic and prognosis markers for lung adenocarcinoma.

In the present study, we analyzed GEO datasets to identify differentially expressed genes (DEGs) in lung adenocarcinoma and inhibitor of DNA binding 2 (ID2) was identified. The specific effects of ID2 overexpression or knockdown on Lung adenocarcinoma cell phenotypes were examined. Gene Ontology (GO), Kyoto Encyclopedia of Genes and Genomes (KEGG) are used to connect the genomic and functional information of DEGs, and the PI3K/AKT signaling pathway was shown to participate in the cellular functions of ID2. The dynamic effects of ID2 and the PI3K/AKT agonist 740Y-P on Lung adenocarcinoma malignant behaviors were investigated. Finally, clinical lung adenocarcinoma and adjacent non-cancerous tissues were collected and the levels of ID2 were examined in tissue samples.

\section{Materials And Methods}

\section{Cell lineage and cell culture}


Human lung epithelial cell line, BEAS-2B (CRL-9609), was obtained from ATCC (Manassas, VA, USA) and cultured using a Bronchial Epithelial Cell Growth Medium Bullet Kit (Catalog No. CC-3170, Lonza, Basel, Switzerland). Human Lung adenocarcinoma cell lines, A549 (CCL-185) was obtained from ATCC and cultured in F-12K Medium (Gibco, Waltham, MA, USA) added with 10\% FBS (Invitrogen, Waltham, MA, USA). H1975 and HCC827 was obtained from ATCC (CRL-5908 and CRL-2868) and cultured in RPMI1640 medium (Gibco) added with $10 \% \mathrm{FBS}$ (Invitrogen). All the cells were cultured at $37^{\circ} \mathrm{C}$ in $5 \% \mathrm{CO}_{2}$. For the PI3K/AKT/mTOR signaling activation, cells were incubated with the agonist 740Y-P $(30 \mu \mathrm{M})$ for $24 \mathrm{~h}$.

\section{Cell transfection}

ID2 overexpression was achieved by transfecting ID2-overexpressing vector (ID2 OE) based on pLVX-puro plasmid. Short hairpin RNA cloned into pLVX-shRNA2 plasmid to against ID2 (sh-ID2) was transfected for ID2 knockdown. Blank vector (vector) and non-targeting sequence (sh-NC) were used as negative control, respectively. All transfections were performed with the help of Lipofectamine 3000 Reagent (Thermo Fisher Scientific, Waltham, MA, USA).

\section{Immunoblotting}

The cells and tissues were lyzed in RIPA buffer with ultrasonic probe. Use the bicinchoninic acid protein assay (BCA, Beyotime, China) was applied for determining the concentration of the protein samples. Protein samples (40 $\mu \mathrm{g}$ per lane) were separated using the Tris-glycine SDS-PAGE ( $8 \%$ to $15 \%$ ) and then transferred onto PVDF membranes (Sigma-Aldrich, St. Louis, MO, USA). After blocking the non-specific bindings, the membranes were incubated with the following primary antibodies: ID2 (ab90055, Abcam, Cambridge, MA, USA), N-cadherin (ab76011, Abcam), E-cadherin (20874-1-AP, Proteintech, Wuhan, China), MMP2 (ab92536, Abcam), MMP9 (CSB-PA058909, Cusabio, Wuhan, China), AKT (Y409094, ABM, Vancouver, Canada), p-AKT (66444-1-1g, Proteintech), mTOR (ab2732, Abcam), p-mTOR (ab109268, Abcam) overnight at $4^{\circ} \mathrm{C}$. Then, the primary antibody was detected with goat anti-mouse antibody conjugated with horseradish peroxidase. Enhanced Chemiluminescence Kit (ECL, Pierce) was used for signal visualization.

\section{Cell Counting Kit 8 (CCK-8) assay}

Use a CCK-8 kit (Sigma-Aldrich) to evaluate cell viability. Target cells were plated in the 96 -well plates at a density of $5 \times 10^{3}$ cells/well and incubated for $24 \mathrm{~h}$. After $48 \mathrm{~h}$ of transfection. $200 \mu \mathrm{l}$ of CCK-8 solution was added to each well and incubated for another $3 \mathrm{~h}$ at $37^{\circ} \mathrm{C}$. At the end of the incubation, cells were washed and the optical density (OD) was examined at $450 \mathrm{~nm}$ using a microplate reader (Thermo Fisher Scientific).

\section{Colony formation}

Cells, transfected or non-transfected, were cultured in a 6-well plate with standard culture medium overnight. Fourteen days later, all the cells were fixed with methanol and stained with crystal violet $(0.1 \%)$. 
Finally, count the colonies under an optical microscope.

\section{Transwell for cell migration}

Transwell permeable support (8- $\mu \mathrm{m}$, Corning, Tewkesbury, USA) was used for cell migration. Cells, transfected for $48 \mathrm{~h}$ or non-transfected, were suspended in serum-free medium into the upper chamber, culture medium containing $10 \%$ FBS was added to the lower chamber. After $24 \mathrm{~h}$ of incubation, the cells stayed on the upper surface of the membrane (non-invasive or non-migrating) were removed. Cells on the bottom surface of the membrane (migratory) were fixed with 4\% paraformaldehyde for $10 \mathrm{~min}$, and stained with $0.4 \%$ crystal violet solution. The migratory cells were imaged using a digital microscopy (Olympus, Tokyo, Japan).

\section{Clinical sampling}

A total of 15 paired Lung adenocarcinoma and adjacent non-cancerous tissues were obtained from patients who underwent lung cancer surgery in Xiangya Hospital, of Central South University. All the samples were immediately stored in a liquid nitrogen tank for long-term preservation. All patients included in this work or their relatives signed informed consent forms. This study was approved by the Ethics Committee of the Xiangya Hospital, of Central South University.

\section{Histopathological examination}

After section, tissue samples were placed in 10\% paraformaldehyde and fixed overnight, processed, and embedded in paraffin. Tissues were then cut into 4- $\mu \mathrm{m}$ slices, dewaxed, and applied for Hematoxylin and eosin (H\&E) staining or Immunohistochemical (IHC) staining. For IHC staining, sections were incubated with anti-ID2 (ab90055, Abcam) for $1 \mathrm{~h}$ at $37^{\circ} \mathrm{C}$, then incubated with biotinylated goat anti-mouse IgG secondary antibody (Boster, China) for $20 \mathrm{~min}$. SABC (streptavidin-biotin complex) was added to the samples and incubated at $37^{\circ} \mathrm{C}$ for $20 \mathrm{~min}$. $3^{\prime}$-diaminobenzidine (DAB) color was permitted to develop for 5 min and samples were counterstained using hematoxylin. The slices visualized and quantified using an Olympus microscope.

\section{Statistics analysis}

At least three parallel experiments were performed for each experiment. All data were processed using the GraphPad software (San Diego, CA, USA) and shown as mean \pm standard deviation (S.D.). One-way analysis of variance (ANOVA) followed by a Tukey's multiple comparison test or Student's $t$-test were used to analyze the statistical significance of the differences. $P$ value $<0.05$ is considered statistically significant.

\section{Results}

Selecting differentially expressed genes associated with lung adenocarcinoma carcinogenesis 
First, GSE116959, GSE130779, GSE118370 and GSE43458 were downloaded and analyzed for differentially-expressed genes between lung adenocarcinoma and adjacent tissues, and a total of 97 genes were identified differentially expressed in all the four datasets $(|\log F C|>1, p<0.05)$ (Fig.S1A). Gene Ontology (GO) of those 97 genes found that gene functions were enriched in proliferation of epithelial tissue, abnormal expansion of metastatic pathways, lung development (Fig.S1B). Kyoto Encyclopedia of Genes and Genomes (KEGG) found that these 97 genes were significantly enriched in the pathways of tumor proteoglycan, cytochrome, calcium signaling (Fig.S1C). Among these genes, ID2 extracted our attention because of the critical role of the ID family in lung adenocarcinoma carcinogenesis [24, 25]. More importantly, ID2 expression is associated with the survival in patients with lung adenocarcinoma from TCGA-LUAD (Fig.S2A), ID2 expression is more downregulated in lung adenocarcinoma from advanced TNM stages (Fig.S2B-D). As a further confirmation, Fig.1 showed the aberrant downregulation of ID2 in lung adenocarcinoma tissues compared with adjacent normal tissues, according to TCGA-LUAD database (Fig.1A), GSE32863 (Fig.1B), GSE11969 (Fig.1C), GSE10072 (Fig.1D), GSE43458 (Fig.1E), and GSE85841 (Fig.1F). Thus, ID2 might play a role in lung adenocarcinoma carcinogenesis.

\section{ID2 overexpression and knockdown functions on lung adenocarcinoma cell phenotypes}

To explore the specific effects of ID2 on lung adenocarcinoma cell phenotypes, we examined the protein levels of ID2 in a normal cell line, BEAS-2B, and three lung adenocarcinoma cell lines, A549, H1975, and HCC827 for selecting proper cell models, Fig.2A shows that ID2 protein levels were markedly decreased in A549 and H1975 cells compared with those in BEAS-2B cells. Thus, ID2 overexpression or knockdown were conducted in A549 and H1975 cells by the transfection of ID2 OE (compared with vector) or sh-ID2 (compared with sh-NC). Transfection efficiency in both cell lines was verified by Immunoblotting (Fig.2B). In both A549 and H1975 cell lines, ID2 overexpression remarkably suppressed cell viability (Fig.2C), colony formation capacity (Fig.2D), and cell migration (Fig.2E), on the contrary, ID2 knockdown promoted the malignant behaviors of both cell lines (Fig.2C-E). Consistently, ID2 overexpression increased the protein levels of epithelial marker E-cadherin and decreased N-cadherin, MMP2, and MMP9 (Fig.2F), whereas ID2 knockdown conversely modulated these proteins (Fig.2F). Since co-expressed genes of ID2 were enriched in the PI3K/AKT signaling, we also examined the changes in the signaling. As shown in Fig.2G, ID2 overexpression blocked, whereas ID2 knockdown promoted the phosphorylation of AKT and mTOR.

\section{ID2 modulates lung cancer cell phenotypes through the PI3KJAKT signaling}

Since ID2 induces changes in the PI3K/AKT signaling, next, we investigated whether the signaling participates in ID2 cellular functions on lung cancer cells. Target cells were transfected with ID2 OE for 48 $\mathrm{h}$, treated with $30 \mu \mathrm{M} 740 \mathrm{Y}-\mathrm{P}$ for $24 \mathrm{~h}$, and examined for related indexes. Similarly, ID2 overexpression inhibited cell viability (Fig.3A), colony formation capacity (Fig.3B), and cell migration (Fig.3C), whereas 740Y-P treatment promoted the malignant behaviors of both cell lines (Fig.3A-C), the effects of ID2 overexpression on lung cancer cell phenotypes were partially reversed by 740Y-P treatment (Fig.3A-C). Consistently, opposite to ID2 overexpression effects, 740Y-P treatment decreased the protein levels of 
epithelial marker E-cadherin and increased N-cadherin, MMP2, and MMP9 (Fig.3D), and partially reversed the effects of ID2 overexpression on these proteins (Fig.3D). As for the PI3K/AKT signaling, ID2 overexpression inhibited, whereas 740Y-P treatment promoted the phosphorylation of AKT and mTOR, also, the effects of ID2 overexpression on AKT and mTOR phosphorylation were partially reversed by 740Y-P treatment (Fig.3E).

\section{ID2 levels in clinical tissue samples}

Finally, we collected clinical lung adenocarcinoma and adjacent non-cancerous tissues to examine the levels of ID2 in tissue samples. The histopathological features of collected samples were confirmed by H\&E staining (Fig.4A). IHC staining showed that ID2 content was reduced in lung adenocarcinoma tissue samples (Fig.4B), which was further evidenced by Immunoblotting that the protein levels of ID2 were remarkably decreased in lung adenocarcinoma tissues compared with the control group (Fig.4C). Similarly, the mRNA expression level of ID2 was downregulated in lung adenocarcinoma tissues compared with the control group (Fig.4D).

\section{Discussion}

Herein, we identified ID2 as an aberrantly downregulated gene in lung adenocarcinoma. ID2 overexpression suppressed lung adenocarcinoma cell viability, colony formation capacity, and migration. ID2 overexpression also reduced the protein levels of N-cadherin, MMP2, MMP9, and the phosphorylation of AKT and mTOR. The PI3K/AKT/mTOR signaling agonist exerted opposite effects on lung adenocarcinoma cells to those of ID2 overexpression, and partially reversed the effects of ID2 overexpression. In tissue samples, ID2 protein levels and mRNA expression were also downregulated compared with those in adjacent non-cancerous tissues.

ID2 is a member of the basic helix-loop-helix protein family [26, 27]. ID2 could modulate cell proliferation and differentiation and tumor development and carcinogenesis [28-30]. Reportedly, ID2 overexpression can antagonize EMT induced by TGF- $\beta$, thus exerting a tumor-suppressive effect on normal mouse mammary epithelial cells, mouse lens epithelial cells, and human renal proximal tubule epithelial cells [31-33]. In hepatocellular carcinoma, ID2 expression was significantly downregulated; ID2 suppressed hepatocellular carcinoma metastasis by regulating EMT processes [34]. Elevated ID2 protein is related to better prognosis of patients with primary breast cancer, and could suppress breast cancer cell invasion [35]. Similarly, herein, we identified the aberrant downregulation of ID2 in lung adenocarcinoma; high expression of ID2 is a protective factor for lung adenocarcinoma patients' overall survival. Regarding the specific cellular functions, in breast cancer, cytoplasmic ID2 protein is correlated to suppressed cell invasion and less aggressive tumor phenotypes [35, 36]. Also, in breast cancer, Wen et al. [37] identified ID2 as an inhibitor of the EMT through upregulating Notch3. In lung adenocarcinoma cells, ID2 overexpression inhibited cell viability, colony formation, and migration, indicating that ID2 also serves as a tumor-suppressor in lung adenocarcinoma. 
To further understand the mechanism underlying the tumor-suppressive role of ID2 in lung adenocarcinoma, we constructed co-expression network of ID2 and performed signaling enrichment annotation; co-expressed genes of ID2 were significantly enriched in the PI3K/AKT/mTOR signaling pathway. Multiple studies have shown up to $50-70 \%$ AKT phosphorylation in NSCLC, which indicates that the over-activation of the PI3K/AKT/mTOR signaling in NSCLC is a common event [38-40]. The upregulation of the mTOR pathway has also been confirmed in a considerable proportion of NSCLC, and pmTOR has increased in up to $90 \%$ of adenocarcinoma patients [42-44]. The PI3K/AKT/mTOR signaling is also implicated in autophagy [45], proliferation, and apoptosis [46] of lung adenocarcinoma cells. In the present study, ID2 overexpression remarkably hindered AKT and mTOR phosphorylation, suggesting that ID2 might exert the tumor-suppressive functions through modulating the PI3K/AKT/mTOR signaling.

Considering the frequent over-activation of the PI3K/AKT/mTOR signaling in cancers, the signaling agonist 740Y-P commonly plays an oncogenic role, modulating cancer cell growth, apoptosis, and migration. In SASH1-overexpressed HepG2 and HCCLM3 cells, 740Y-P treatment significantly promoted cell migration and invasion [47]. In ovarian cancer, 740Y-P treatment attenuated the inhibitory effects of circRHOBTB3 overexpression on cancer cell proliferation, metastasis and glycolysis [48]. In NSCLC, 740Y$\mathrm{P}$ treatment also reversed IncRNA TBX5-AS1 blockage of cancer cell colony formation, migration, and invasion [49]. In the present study, 740Y-P treatment on ID2-overexpressed lung adenocarcinoma cells also significantly attenuated ID2 overexpression blockage of cancer cell viability, colony formation, and migration. In addition, consistent with the above-mentioned studies, 740Y-P treatment promoted the phosphorylation of AKT and mTOR.

\section{Conclusions}

Altogether, these findings indicate that ID2 exerts its tumor-suppressive effects on lung adenocarcinoma cell malignant behaviors through inhibiting the activation of the PI3K/AKT/mTOR signaling pathway. Restoring ID2 expression in lung adenocarcinoma cells might improve the curative effect of lung cancer therapies.

\section{Abbreviations}




\begin{tabular}{|ll|}
\hline DEGs & differentially expressed genes \\
\hline GO & Gene Ontology \\
\hline KEGG & Kyoto Encyclopedia of Genes and Genomes \\
\hline ID2 & Inhibitor of DNA binding 2 \\
\hline NSCLC & non-small-cell lung cancer \\
\hline GEO & Gene Expression Comprehensive Database \\
\hline TCGA & The Cancer Genome Atlas \\
\hline BCA & bicinchoninic acid protein assay \\
\hline OD & optical density \\
\hline H\&E & Hematoxylin and eosin \\
\hline IHC & Immunohistochemical \\
\hline
\end{tabular}

\section{Declarations}

Ethics approval and consent to participate

This paper were approved by the Medical Ethics Committee of Xiangya Hospital of Central South University. Approval ID:202105089.

Consent for publication

Not applicable

Availability of data and materials

All data generated or analysed during this study are included in this published article [and its supplementary information files].

\section{Conflict of Interest}

None

\section{Funding}

This study was supported by Natural Science Foundation of Hunan Province (2020JJ5897).

Authors' contributions 
P-WZ and LY designed the research and wrote the manuscript. $\mathrm{CJ}$ and $\mathrm{H}-\mathrm{RX}$ performed the majority of experiments. T-YJ and JJ collected and analyzed the clinical experimental data, performed the figures and the statistical analyses. All the authors read and approved the final manuscript.

\section{Acknowledgements}

Not applicable

\section{References}

1. Arbyn, M., et al., Estimates of incidence and mortality of cervical cancer in 2018: a worldwide analysis. Lancet Glob Health, 2020. 8(2): p. e191-e203.

2. Sher, T., G.K. Dy, and A.A. Adjei, Small cell lung cancer. Mayo Clin Proc, 2008. 83(3): p. 355-67.

3. Noguchi, M., et al., Small adenocarcinoma of the lung. Histologic characteristics and prognosis. Cancer, 1995. 75(12): p. 2844-52.

4. Zappa, C. and S.A. Mousa, Non-small cell lung cancer: current treatment and future advances. Transl Lung Cancer Res, 2016. 5(3): p. 288-300.

5. Viktorsson, K., R. Lewensohn, and B. Zhivotovsky, Systems biology approaches to develop innovative strategies for lung cancer therapy. Cell Death Dis, 2014. 5: p. e1260.

6. Nguyen, D.X., et al., WNT/TCF signaling through LEF1 and HOXB9 mediates lung adenocarcinoma metastasis. Cell, 2009. 138(1): p. 51-62.

7. Stewart, D.J., Wnt signaling pathway in non-small cell lung cancer. J Natl Cancer Inst, 2014. 106(1): p. djt356.

8. Borczuk, A.C., et al., Lung adenocarcinoma global profiling identifies type II transforming growth factor-beta receptor as a repressor of invasiveness. Am J Respir Crit Care Med, 2005. 172(6): p. 729 37.

9. Saito, A., et al., The Role of TGF-beta Signaling in Lung Cancer Associated with Idiopathic Pulmonary Fibrosis. Int J Mol Sci, 2018. 19(11).

10. Li, T., et al., Genotyping and genomic profiling of non-small-cell lung cancer: implications for current and future therapies. J Clin Oncol, 2013. 31(8): p. 1039-49.

11. Zhou, C., et al., Erlotinib versus chemotherapy as first-line treatment for patients with advanced EGFR mutation-positive non-small-cell lung cancer (OPTIMAL, CTONG-0802): a multicentre, open-label, randomised, phase 3 study. Lancet Oncol, 2011. 12(8): p. 735-42.

12. Lee, J.J., et al., Tracing Oncogene Rearrangements in the Mutational History of Lung Adenocarcinoma. Cell, 2019. 177(7): p. 1842-1857 e21.

13. Lynch, T.J., et al., Activating mutations in the epidermal growth factor receptor underlying responsiveness of non-small-cell lung cancer to gefitinib. N Engl J Med, 2004. 350(21): p. 2129-39.

14. Shaw, A.T., et al., Clinical features and outcome of patients with non-small-cell lung cancer who harbor EML4-ALK. J Clin Oncol, 2009. 27(26): p. 4247-53. 
15. Makinoshima, H., et al., Signaling through the Phosphatidylinositol 3-Kinase (PI3K)/Mammalian Target of Rapamycin (mTOR) Axis Is Responsible for Aerobic Glycolysis mediated by Glucose Transporter in Epidermal Growth Factor Receptor (EGFR)-mutated Lung Adenocarcinoma. J Biol Chem, 2015. 290(28): p. 17495-504.

16. Wang, D.Y., et al., PEST-containing nuclear protein regulates cell proliferation, migration, and invasion in lung adenocarcinoma. Oncogenesis, 2019. 8(3): p. 22.

17. Su, C., et al., Screening of Hub Gene Targets for Lung Cancer via Microarray Data. Comb Chem High Throughput Screen, 2020.

18. Jin, X., et al., Bioinformatics Analysis of Microarray Datasets to Identify Prognostic Factors in Lung Adenocarcinoma. DNA Cell Biol, 2020. 39(6): p. 965-974.

19. Su, H., et al., Identification of potential biomarkers of lung adenocarcinoma brain metastases via microarray analysis of cDNA expression profiles. Oncol Lett, 2019. 17(2): p. 2228-2236.

20. Wu, Q., et al., Identification of novel biomarkers and candidate small molecule drugs in non-small-cell lung cancer by integrated microarray analysis. Onco Targets Ther, 2019. 12: p. 3545-3563.

21. Liu, X.X., et al., A two-circular RNA signature as a noninvasive diagnostic biomarker for lung adenocarcinoma. J Transl Med, 2019. 17(1): p. 50.

22. Wang, $\mathrm{K}$., et al., Identification of differentially expressed genes in non-small cell lung cancer. Aging (Albany NY), 2019. 11(23): p. 11170-11185.

23. He, F., et al., Microarray profiling of differentially expressed IncRNAs and mRNAs in lung adenocarcinomas and bioinformatics analysis. Cancer Med, 2020. 9(20): p. 7717-7728.

24. Lu, X., et al., Prognostic effects of the expression of inhibitor of DNA-binding family members on patients with lung adenocarcinoma. Oncol Lett, 2020. 20(5): p. 143.

25. Zhang, S., et al., Landscape of transcriptional deregulation in lung cancer. BMC Genomics, 2018. 19(1): p. 435.

26. Biggs, J., E.V. Murphy, and M.A. Israel, A human Id-like helix-loop-helix protein expressed during early development. Proc Natl Acad Sci U S A, 1992. 89(4): p. 1512-6.

27. Ellmeier, W., et al., Mutually exclusive expression of a helix-loop-helix gene and N-myc in human neuroblastomas and in normal development. EMBO J, 1992. 11(7): p. 2563-71.

28. Norton, J.D., ID helix-loop-helix proteins in cell growth, differentiation and tumorigenesis. J Cell Sci, 2000. 113 (Pt 22): p. 3897-905.

29. Ling, F., B. Kang, and X.H. Sun, Id proteins: small molecules, mighty regulators. Curr Top Dev Biol, 2014. 110: p. 189-216.

30. Zebedee, Z. and E. Hara, Id proteins in cell cycle control and cellular senescence. Oncogene, 2001. 20(58): p. 8317-25.

31. Saika, S., et al., Adenoviral gene transfer of BMP-7, Id2, or Id3 suppresses injury-induced epithelial-tomesenchymal transition of lens epithelium in mice. Am J Physiol Cell Physiol, 2006. 290(1): p. C2829. 
32. Veerasamy, M., M. Phanish, and M.E. Dockrell, Smad mediated regulation of inhibitor of DNA binding 2 and its role in phenotypic maintenance of human renal proximal tubule epithelial cells. PLoS One, 2013. 8(1): p. e51842.

33. Gervasi, M., et al., JunB contributes to Id2 repression and the epithelial-mesenchymal transition in response to transforming growth factor-beta. J Cell Biol, 2012. 196(5): p. 589-603.

34. Zhou, Y., et al., LncRNA ID2-AS1 suppresses tumor metastasis by activating the HDAC8/ID2 pathway in hepatocellular carcinoma. Cancer Lett, 2020. 469: p. 399-409.

35. Stighall, M., et al., High ID2 protein expression correlates with a favourable prognosis in patients with primary breast cancer and reduces cellular invasiveness of breast cancer cells. Int J Cancer, 2005.

115(3): p. 403-11.

36. Itahana, Y., et al., Role of Id-2 in the maintenance of a differentiated and noninvasive phenotype in breast cancer cells. Cancer Res, 2003. 63(21): p. 7098-105.

37. Wen, X.F., et al., Inhibitor of DNA Binding 2 Inhibits Epithelial-Mesenchymal Transition via UpRegulation of Notch3 in Breast Cancer. Transl Oncol, 2018. 11(5): p. 1259-1270.

38. Balsara, B.R., et al., Frequent activation of AKT in non-small cell lung carcinomas and preneoplastic bronchial lesions. Carcinogenesis, 2004. 25(11): p. 2053-9.

39. Tsurutani, J., et al., Evaluation of two phosphorylation sites improves the prognostic significance of Akt activation in non-small-cell lung cancer tumors. J Clin Oncol, 2006. 24(2): p. 306-14.

40. Cappuzzo, F., et al., Prospective study of gefitinib in epidermal growth factor receptor fluorescence in situ hybridization-positive/phospho-Akt-positive or never smoker patients with advanced non-smallcell lung cancer: the ONCOBELL trial. J Clin Oncol, 2007. 25(16): p. 2248-55.

41. Malanga, D., et al., Activating E17K mutation in the gene encoding the protein kinase AKT1 in a subset of squamous cell carcinoma of the lung. Cell Cycle, 2008. 7(5): p. 665-9.

42. Dobashi, Y., et al., Critical and diverse involvement of Akt/mammalian target of rapamycin signaling in human lung carcinomas. Cancer, 2009. 115(1): p. 107-18.

43. Dobashi, Y., et al., Paradigm of kinase-driven pathway downstream of epidermal growth factor receptor/Akt in human lung carcinomas. Hum Pathol, 2011. 42(2): p. 214-26.

44. Hiramatsu, M., et al., Activation status of receptor tyrosine kinase downstream pathways in primary lung adenocarcinoma with reference of KRAS and EGFR mutations. Lung Cancer, 2010. 70(1): p. 94102.

45. Chen, J., et al., Regulation of different components from Ophiopogon japonicus on autophagy in human lung adenocarcinoma A549Cells through PI3K/Akt/mTOR signaling pathway. Biomed Pharmacother, 2017. 87: p. 118-126.

46. Gu, X., et al., Human Schlafen 5 Inhibits Proliferation and Promotes Apoptosis in Lung Adenocarcinoma via the PTEN/PI3K/AKT/mTOR Pathway. Biomed Res Int, 2021. 2021: p. 6628682.

47. Sun, C., et al., Involvement of PI3K/Akt pathway in the inhibition of hepatocarcinoma cell invasion and metastasis induced by SASH1 through downregulating Shh-Gli1 signaling. Int J Biochem Cell 
Biol, 2017. 89: p. 95-100.

48. Yalan, S., et al., Circular RNA circRHOBTB3 inhibits ovarian cancer progression through PI3K/AKT signaling pathway. Panminerva Med, 2020.

49. Qu, Q.H., S.Z. Jiang, and X.Y. Li, LncRNA TBX5-AS1 Regulates the Tumor Progression Through the PI3K/AKT Pathway in Non-Small Cell Lung Cancer. Onco Targets Ther, 2020. 13: p. 7949-7961.

\section{Figures}

A

TCGA-LUAD

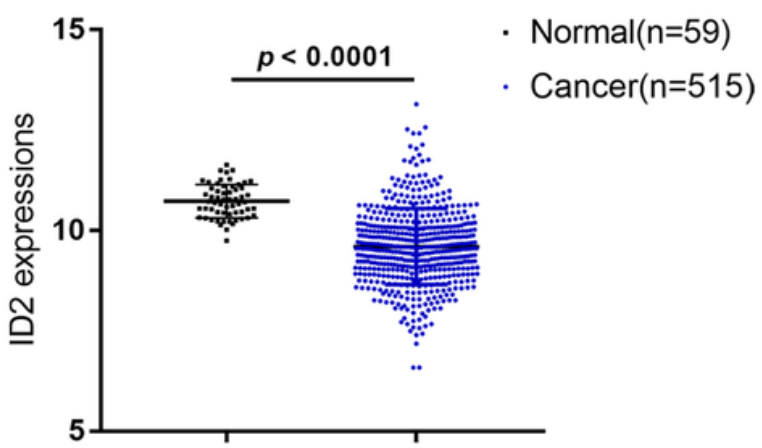

C

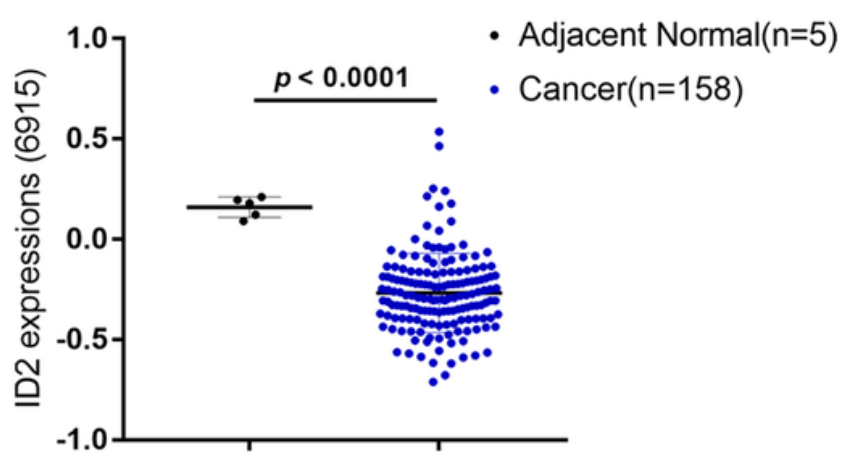

$E$

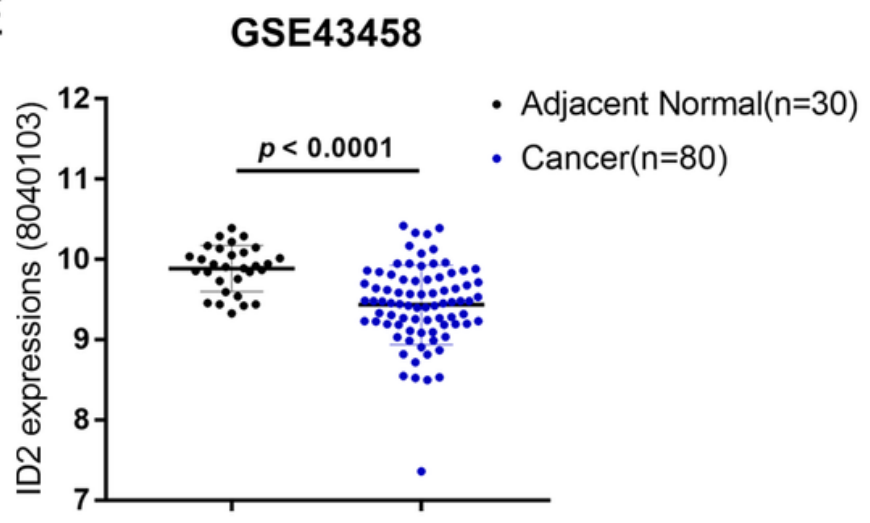

B

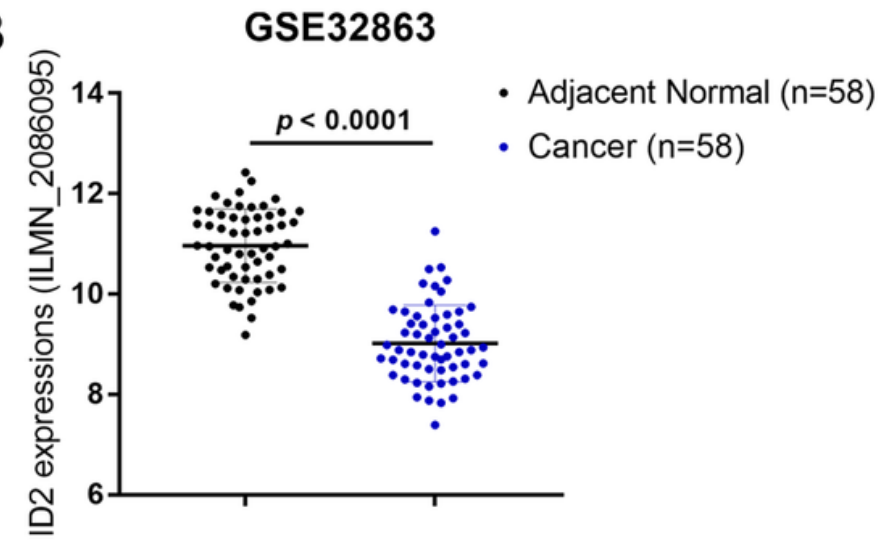

D

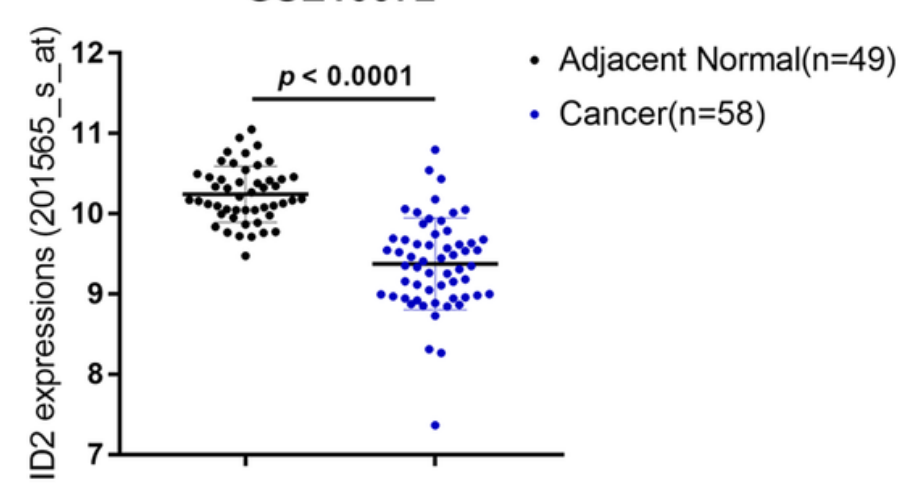

F

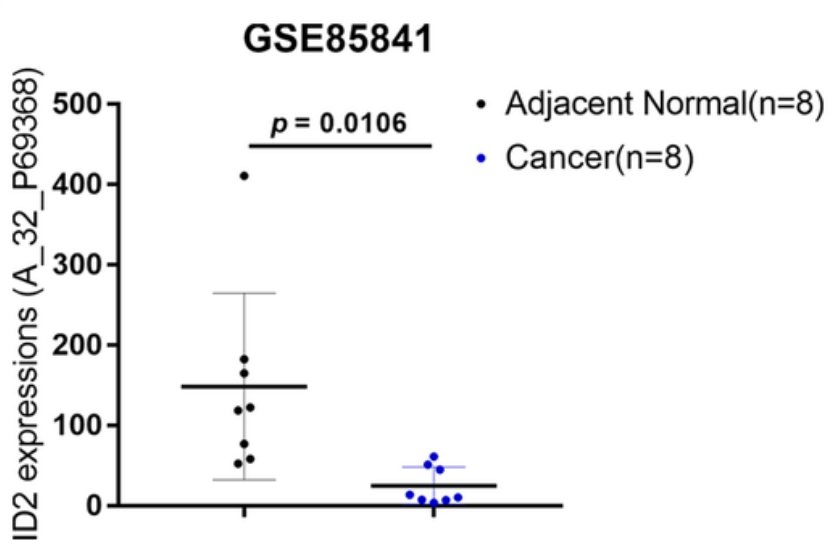

Figure 1 
Aberrant downregulation of ID2 in lung adenocarcinoma tissues The expression levels of ID2 in lung adenocarcinoma and adjacent normal tissues, according to TCGA-LUAD database (A), GSE32863 (B), GSE11969 (C), GSE10072 (D), GSE43458 (E), and GSE85841 (F).
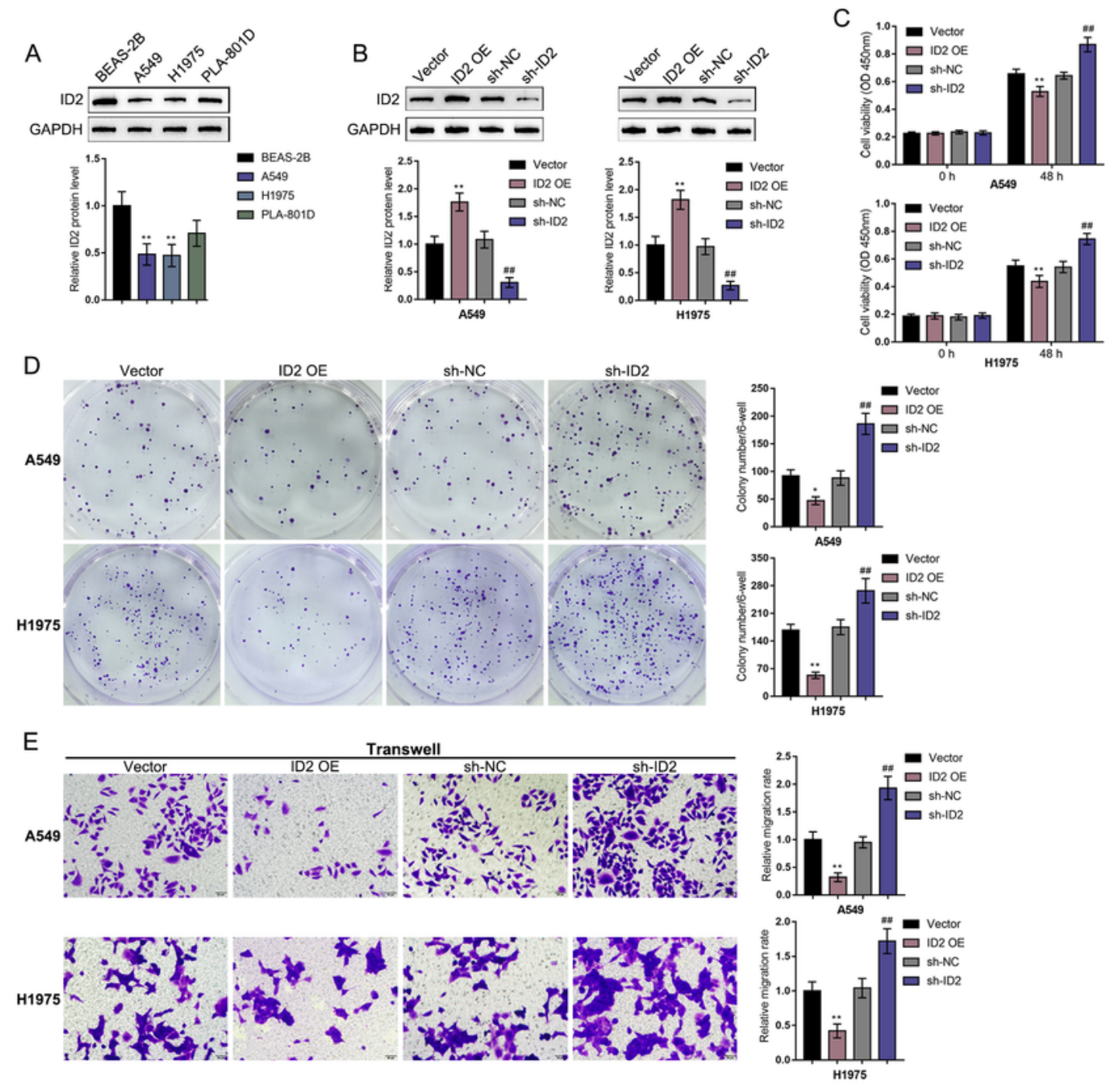

F

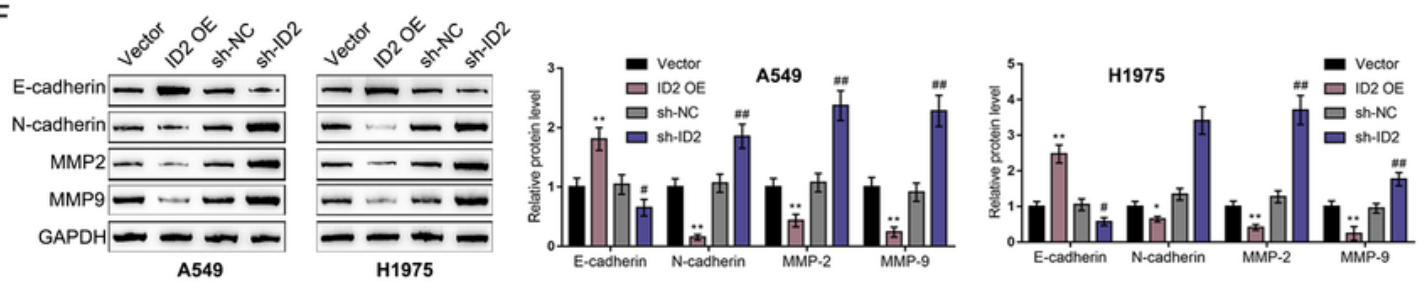

G
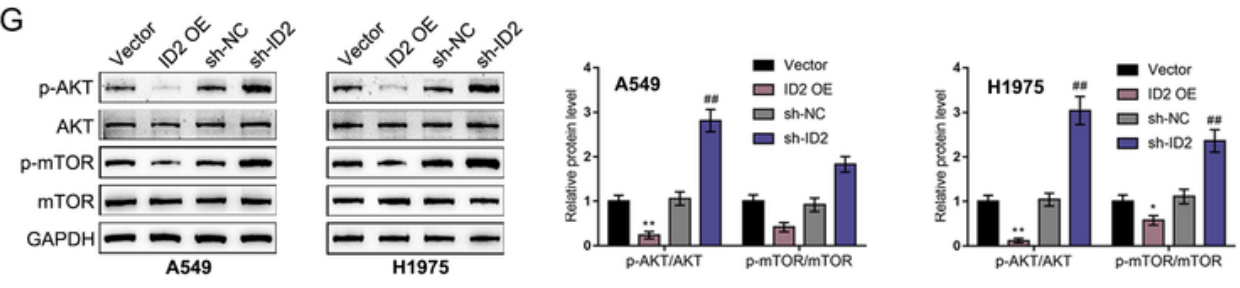

Figure 2

Effects of ID2 overexpression and knockdown on lung adenocarcinoma cell phenotypes (A) The protein levels of ID2 were examined in a human lung epithelial cell line, BEAS-2B, and three lung adenocarcinoma 
cell lines, A549, H1975, and HCC827 by Immunoblotting. (B) ID2 overexpression or knockdown was achieved in A549 and H1975 cells by transfecting ID2 OE (compared with vector) or sh-ID2 (compared with sh-NC). The overexpression or knockdown of ID2 was confirmed by Immunoblotting. Then, A549 and H1975 cells were transfected with ID2 or sh-ID2 and examined for cell viability by CCK-8 assay (C), colony formation capacity by Colony formation assay (D), cell migration by Transwell assay (E), the protein levels of E-cadherin, N-cadherin, MMP2, MMP9, p-AKT, AKT, p-mTOR, and mTOR by Immunoblotting (F-G). $* P<0.05, * * P<0.01, \# P<0.05, \# \# P<0.01$.

A
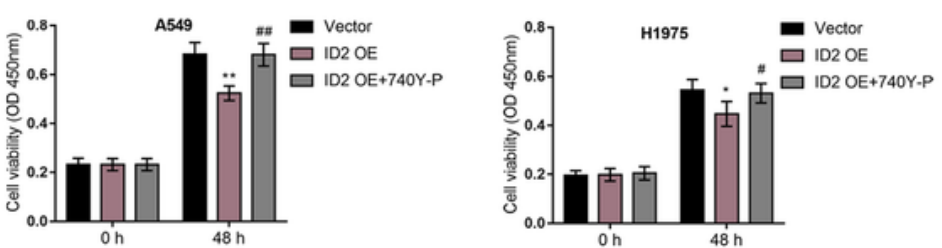

B

ID2 OE

ID2 OE+740Y-P
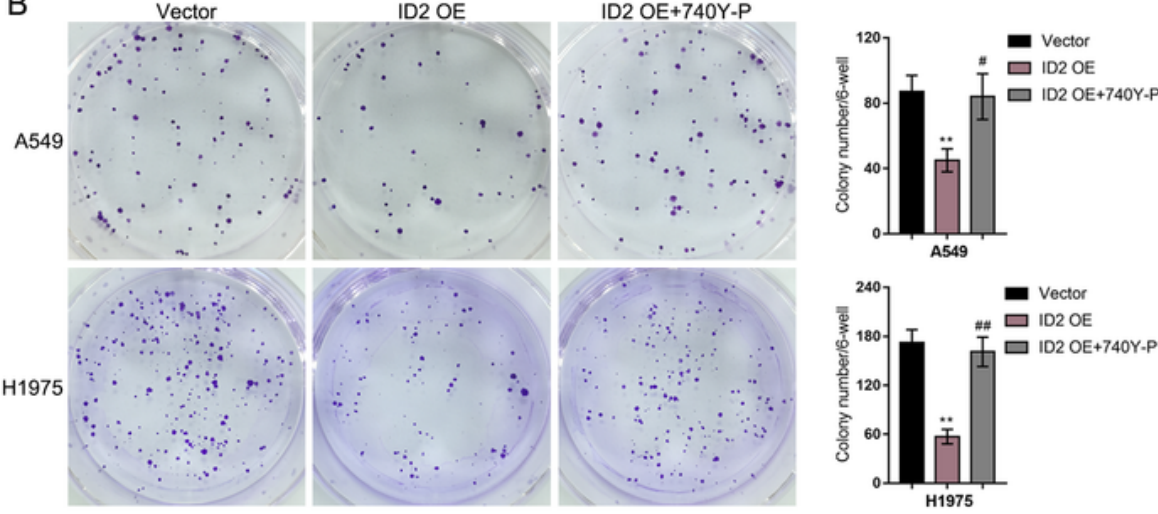

C
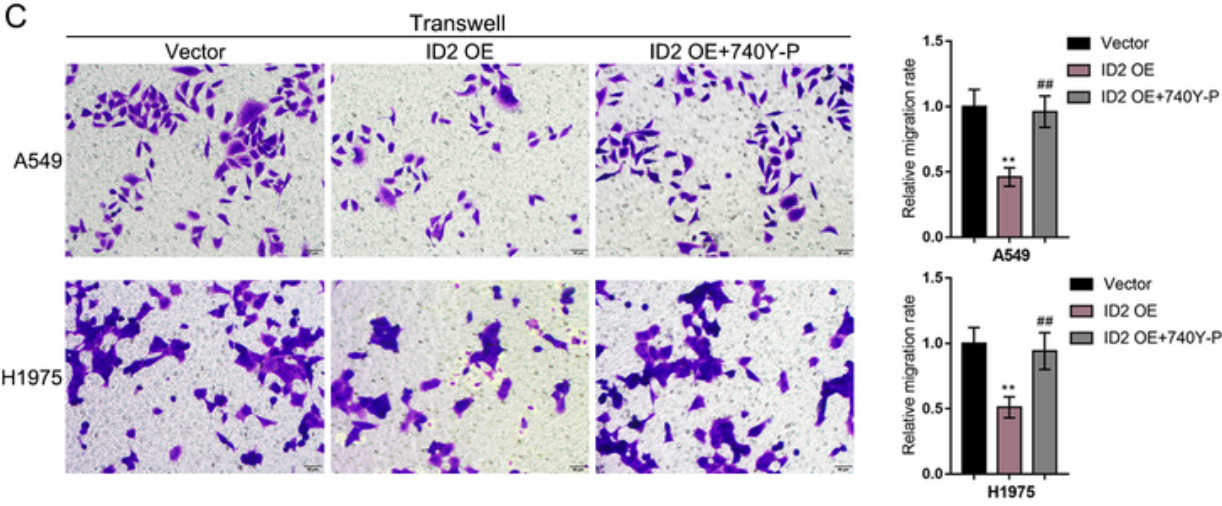

D
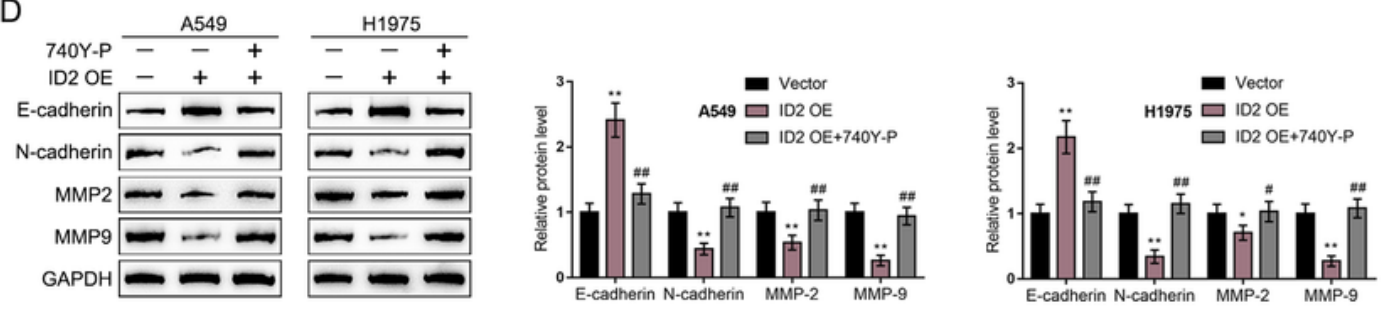

E
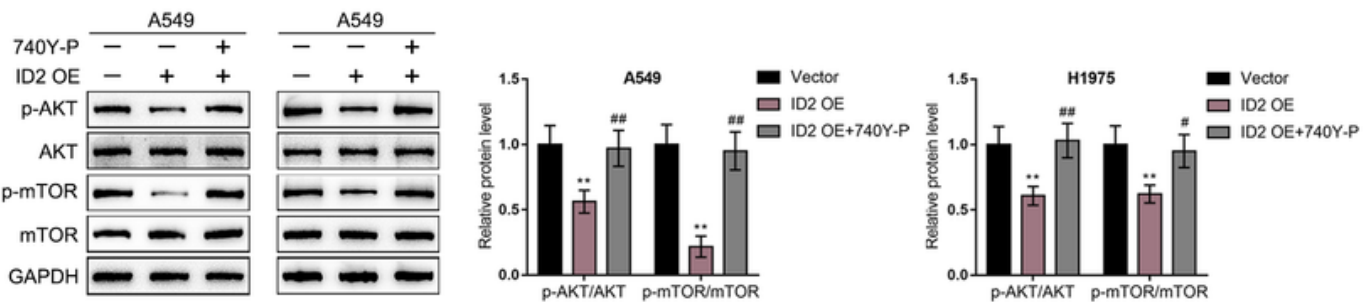


\section{Figure 3}

ID2 modulates lung adenocarcinoma cell phenotypes through the PI3K/AKT signaling Target cells were transfected with ID2 OE for $48 \mathrm{~h}$, treated with $30 \mu \mathrm{M} 740 \mathrm{Y}-\mathrm{P}$ for $24 \mathrm{~h}$, and examined for cell viability by CCK-8 assay (A), colony formation capacity by Colony formation assay (B), cell migration by Transwell assay (C), the protein levels of E-cadherin, N-cadherin, MMP2, MMP9, p-AKT, AKT, p-mTOR, and mTOR by Immunoblotting (D-E). ${ }^{*} \mathrm{P}<0.05,{ }^{*} * \mathrm{P}<0.01, \# \mathrm{P}<0.05$, \#\#P<0.01.

A
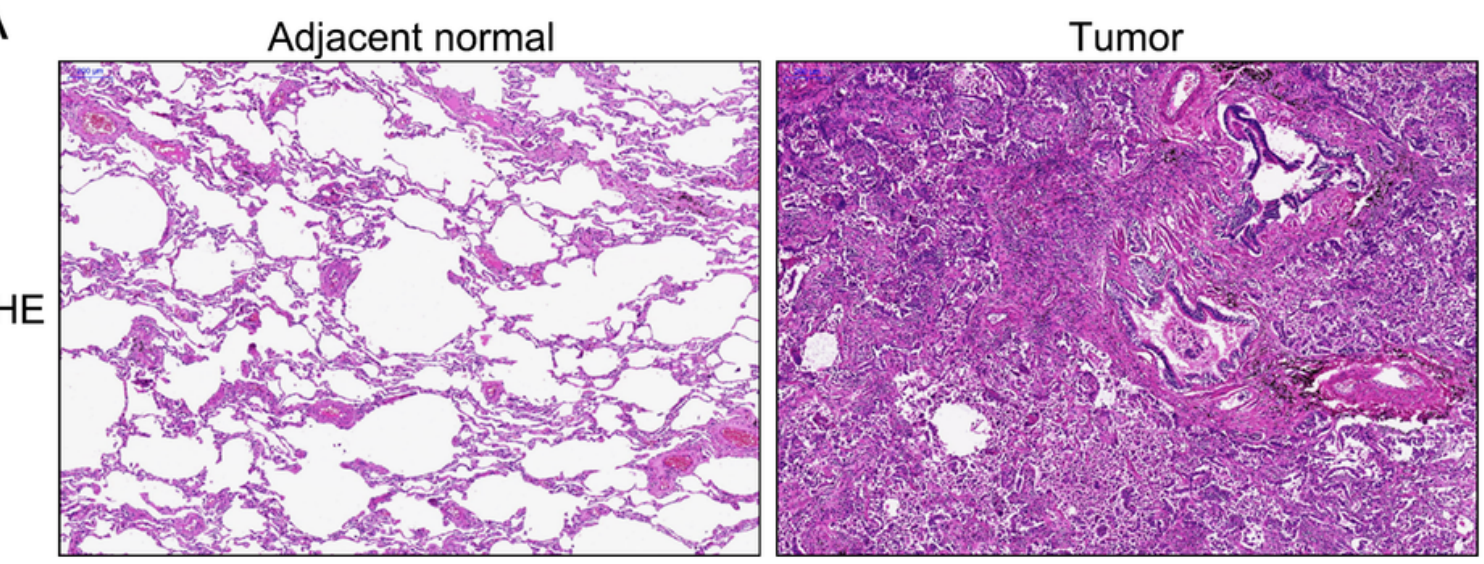

B

Adjacent normal
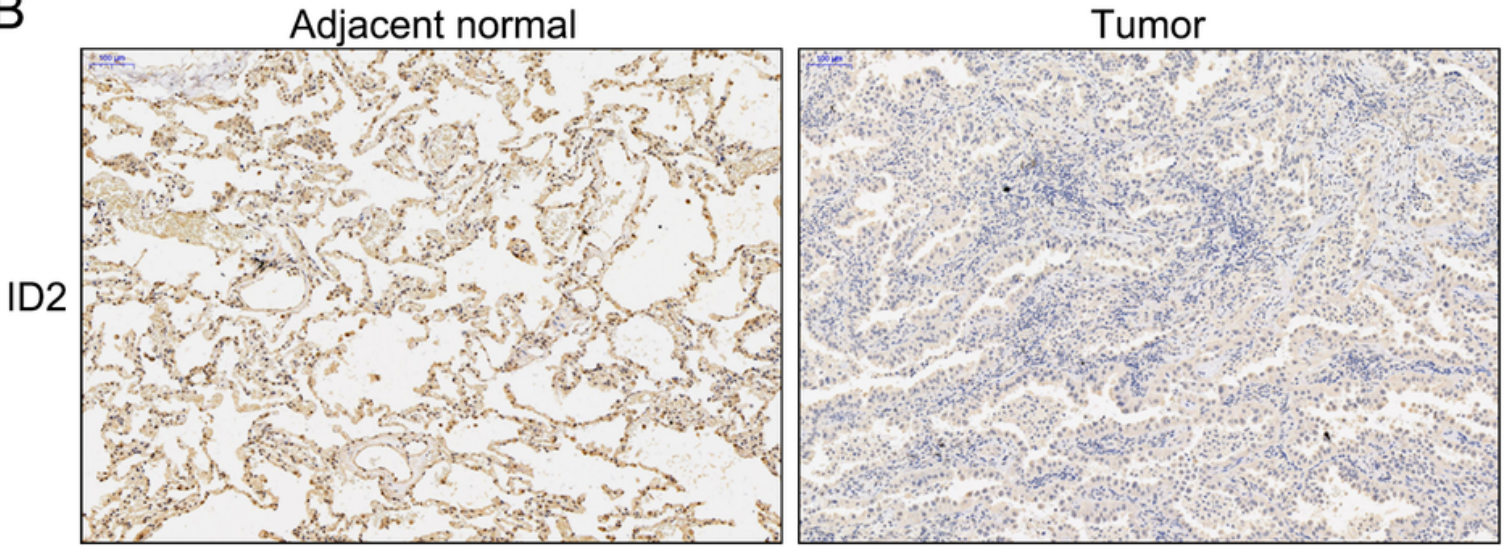

C
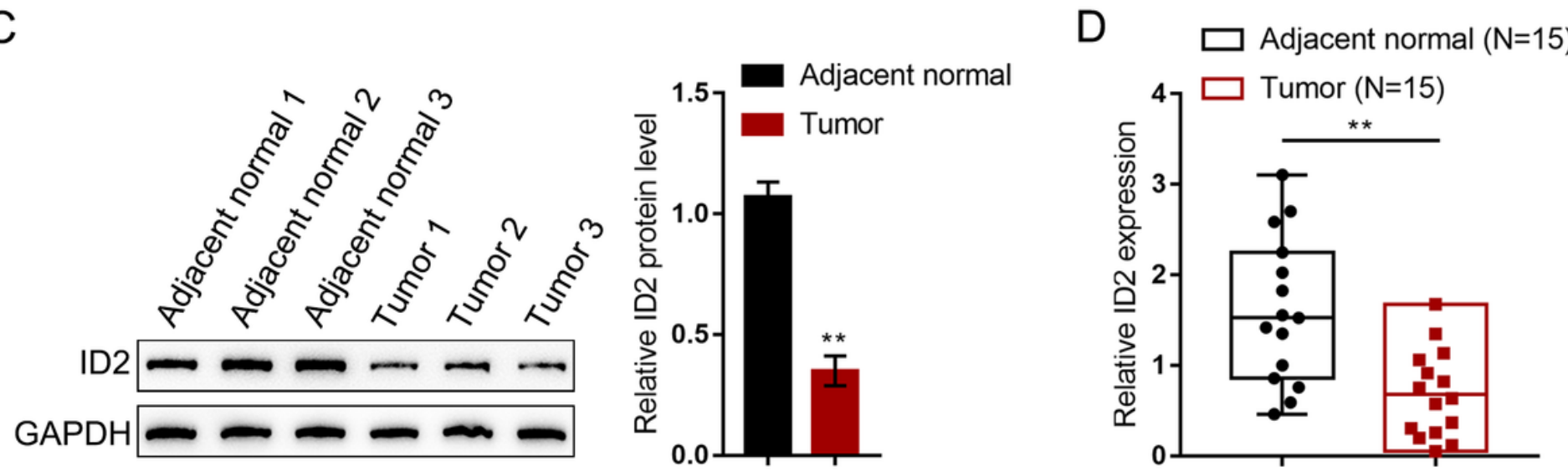

Figure 4 
ID2 levels in clinical tissue samples (A) Clinical lung adenocarcinoma and adjacent non-cancerous tissues were collected and examined for the histopathological features by H\&E staining. (B) The levels of ID2 in tissue samples were examined by Immunohistochemical (IHC) staining. (C) The protein levels of ID2 in tissue samples were examined by Immunoblotting. (D) The mRNA expression levels of ID2 in tissue samples were examined by qRT-PCR. ${ }^{\star \star} \mathrm{P}<0.01$.

\section{Supplementary Files}

This is a list of supplementary files associated with this preprint. Click to download.

- FS1.tif

- FS2.tif 